\title{
Secular changes in dementia risk indices among 70-year-olds: a comparison of two Finnish cohorts born 20 years apart
}

\author{
Jenni Vire ${ }^{1}\left[\right.$ Marika Salminen $^{2,3} \cdot$ Paula Viikari $^{1} \cdot$ Tero Vahlberg $^{4} \cdot$ Seija Arve $^{2} \cdot$ Matti Viitanen $^{1,5} \cdot$ Laura Viikari $^{1}$
}

Received: 2 April 2019 / Accepted: 9 April 2019 / Published online: 4 May 2019

(c) The Author(s) 2019

\begin{abstract}
Aims To compare dementia risk indices among two separate cohorts of 70-year-olds born 20 year apart.

Methods Community-dwelling 70-year-old Finns were examined with similar examinations in $1991(n=1032)$ and in 2011 $(n=960)$. Dementia risk was assessed with the CAIDE Dementia Risk Score (CAIDE) $(n=1516)$, the Brief Dementia Risk Index (BDRI) $(n=1598)$ and the Dementia Screening Indicator (DSI) $(n=1462)$.

Results The proportion of subjects with moderate or high risk for dementia was significantly higher in earlier than in later born cohort according to CAIDE (99\% and 94\%, respectively, $p<0.001)$ and BDRI ( $41 \%$ and 15\%, $p<0.001$ ), but not according to DSI ( $5 \%$ and $6 \%, p=0.184)$. The total scores of the earlier born cohort were significantly higher than those of the later born cohort according to all three indices.

Conclusions According to dementia risk indices, it seems that dementia risk has decreased among community-dwelling 70-year-old subjects during the last decades in Finland.
\end{abstract}

Keywords Dementia $\cdot$ Risk index $\cdot$ Older adults $\cdot$ Cohort comparison

\section{Introduction}

Dementia is a major cause of institutionalization in older people, and therefore, it is a major global public health concern [1]. Although no curative treatment is available, epidemiological studies have provided evidence of modifiable risk and protective factors of dementia [2, 3]. Dementia risk indices are tools that quickly and efficiently combine information on known risk factors of dementia, and thus identify

Jenni Vire

jenni.vire@dnainternet.net

1 Department of Geriatrics, Faculty of Medicine, Turku City Hospital, University of Turku, Kunnallissairaalantie 20, 20700 Turku, Finland

2 City of Turku, Welfare Division, Yliopistonkatu 30, 20101 Turku, Finland

3 Unit of Family Medicine, Faculty of Medicine, University of Turku, Lemminkäisenkatu, 20014 Turku, Finland

4 Institute of Clinical Medicine, Biostatistics, University of Turku, Turku, Finland

5 Division of Clinical Geriatrics Karolinska Institutet, Karolinska University Hospital, Huddinge, 14186 Stockholm, Sweden individuals with a risk for dementia who can be referred for more frequent monitoring and early interventions in order to prevent or delay onset of cognitive decline [4]. The aim of this cross-sectional study was to compare dementia risk indices in two birth cohorts of 70-year-old community-dwelling Finns born 20 years apart.

\section{Material and methods}

\section{Study population}

The data of two 70-year-old cohorts of community-dwelling older people born in 1920 and 1940 and living in the city of Turku, in Southwest Finland, were collected by using similar postal questionnaires, interviews and clinical examinations in 1991 (The Turku Elderly Study) and 2011 (The New Turku Elderly Study). The data on the subjects included in both cohorts were obtained from the central population register. Altogether 1032 subjects from the earlier cohort (70\% of those invited) and 960 subjects from the later cohort (73\% of those invited) returned the postal questionnaires and were considered for inclusion in this study. The protocol of data collection and flow charts of the studies are described 
in detail previously [5]. Participants with missing data of dementia risk indices were excluded.

\section{Dementia Risk indices}

The CAIDE Dementia Risk Score (CAIDE) [6], the Brief Dementia Risk Index (BDRI) [7] and the Dementia Screening Indicator (DSI) [8] were used to compare dementia risk between the cohorts. Slightly modified versions of both CAIDE and BDRI were used; DSI was used as an original.

The CAIDE, a seven-item risk index (range 0-15), includes age, education, gender, blood pressure, body mass index (BMI), total cholesterol, and physical activity [6]. In our study, physical inactivity was defined as not having daily outdoor activities. Those scoring $\geq 6$ points have shown to have an elevated risk for developing dementia during the following 20 years among Finnish middle aged (39-64 years) population [6].

The BDRI [7] consists of 12 items (range 0-14): age, recall of three words presented after a brief delay, copying a figure of two pentagons that intersect to form a diamond, performing either of the first two steps of three-step request, naming at least ten four-legged animals in $30 \mathrm{~s}$, self-reported "trouble keeping my mind on what I was doing" three or more days per week during the past month, medical history of stroke, peripheral artery disease or coronary artery bypass surgery, body mass index and alcohol consumption. We replaced the original item "naming ten four-legged animals in $30 \mathrm{~s}$ " with a mathematic exercise "serial sevens" included in Mini-Mental State Examination. We also included angioplasty in coronary artery bypass surgery. Older subjects (aged $\geq 65$ years) with total scores of $0-2,3-5$ and $\geq 6$ have previously been categorized as having a low, moderate, or high risk for developing dementia during a 6-year follow-up, respectively [7].

The DSI, designed specifically for usage in primary care settings in order to identifying older patients with an increased risk of dementia, includes the following seven items: age, educational attainment, body mass index, presence of diabetes mellitus, history of stroke, need for help in managing money or medications, and depressive symptoms (range $0-56$ ). Subjects scoring $\geq 22$ points have been classified as having a high risk for dementia in 65- to 79-year-olds during a 6-year follow-up [8].

\section{Ethics}

The study protocol was approved by the City of Turku ethical committee on health care and the ethical committee of the Hospital district of Southwest Finland. Informed consent was obtained from all participants.

\section{Statistical analyses}

Differences in dementia risk items and categorized dementia risk level between two cohorts were analyzed by using the Chi-squared test and Fisher's exact test. Differences in mean scores of indices were tested by using two-sample $t$ test. For BDRI and DSI, analyses were also conducted separately for men and women because gender was not included in those indices. $p$ values $<0.05$ were considered statistically significant. All statistical analyses were performed using SAS System for Windows, version 9.4 (SAS Institute Inc., Cary, NC, USA).

\section{Results}

Altogether, 1516, 1598 and 1462 participants (63\% of women) were included in the comparison of dementia risk with CAIDE, BDRI, and DSI between the cohorts, respectively (Table 1). The proportion of subjects with an increased risk for developing dementia was significantly higher in 1920 cohort compared to 1940 cohort according to categorized CAIDE and BDRI but not according to that of DSI. There were distinct differences in proportions of subjects (cohorts combined) categorized as having an increased (moderate or high) risk for developing dementia according to three indices being $96 \%, 27 \%$ and $6 \%$ according to CAIDE, BDRI, and DSI, respectively. The total risk scores of the earlier born cohort were significantly higher than those of the later born cohort according to all three indices.

BDRI and DSI was also analyzed separately in women and men, because gender was not included in either indices. According to BDRI, $38 \%$ and $9 \%(p<0.001)$ of women in 1920 and 1940 cohort, respectively, had moderate or high risk for dementia; corresponding proportions of men were $47 \%$ and $23 \%(p<0.001)$. Also the total scores of BDRI were significantly higher in 1920 cohort compared to 1940 cohort both in women (Mean \pm standard deviation $2.10 \pm 1.36$ and $1.35 \pm 0.92$, respectively $)(p<0.001)$ and in men $(2.41 \pm 1.30$ and $1.84 \pm 1.20)(p<0.001)$. According to DSI, significant difference was found only in total scores among women $(15.06 \pm 3.56$ and $13.68 \pm 5.16)(p \leq 0.001)$, and the difference was in favor of the later cohort.

\section{Discussion}

The results of our study showed that dementia risk, assessed by using dementia risk indices, has decreased among Finnish community-dwelling older adults during the last decades. This is consistent with the evidence of decreasing 
Table 1 Characteristics of CAIDE Dementia Risk Score, the Brief Dementia Risk Index and the Dementia Screening Indicator in 1920 cohort $(n=1032)$ and 1940 cohort $(n=956)$

\begin{tabular}{|c|c|c|c|c|}
\hline & Points & $\begin{array}{l}1920 \text { cohort } \\
n=719 \\
n(\%)\end{array}$ & $\begin{array}{l}1940 \text { cohort } \\
n=797 \\
n(\%)\end{array}$ & $p$ value \\
\hline \multicolumn{5}{|l|}{ CAIDE Dementia Risk Score (CAIDE) } \\
\hline Age $>53$ years & 4 & $719(100)$ & $797(100)$ & 1.000 \\
\hline Education (years) & & & & $<0.001$ \\
\hline$\geq 10$ & 0 & $50(7)$ & $169(21)$ & \\
\hline $7-9$ & 2 & $88(14)$ & $183(23)$ & \\
\hline$<7$ & 3 & $570(79)$ & $445(56)$ & \\
\hline Male & 1 & $230(32)$ & $328(41)$ & $<0.001$ \\
\hline Systolic blood pressure $>140 \mathrm{~mm} \mathrm{Hg}$ & 2 & $513(71)$ & $516(65)$ & 0.006 \\
\hline Body mass index $>30 \mathrm{~kg} / \mathrm{m}^{2}$ & 2 & $115(16)$ & $194(24)$ & $<0.001$ \\
\hline Total cholesterol $>6.5 \mathrm{mmol} / \mathrm{L}$ & 2 & $241(34)$ & $80(10)$ & $<0.001$ \\
\hline Physical inactivity & 1 & $35(5)$ & $27(3)$ & 0.143 \\
\hline Increased risk for dementia & $\geq 6$ & 711 (99) & $746(94)$ & $<0.001$ \\
\hline Total score, mean (SD) & & $9.39(1.75)$ & $8.53(1.99)$ & $<0.001$ \\
\hline The Brief Dementia Risk Index (BDRI) & & $n=704$ & $n=894$ & \\
\hline Age $<75$ years & 0 & $704(100)$ & $894(100)$ & 1.000 \\
\hline Delayed recall, $<2$ of 3 words & 2 & $323(46)$ & $96(11)$ & $<0.001$ \\
\hline Incorrectly copying intersecting pentagons & 1 & $100(14)$ & $56(6)$ & $<0.001$ \\
\hline Incorrectly taking or folding a paper & 1 & $9(1)$ & $5(1)$ & 0.176 \\
\hline Serial seven ${ }^{\mathrm{a}},<3$ of 5 correct & 1 & $73(10)$ & $82(9)$ & 0.422 \\
\hline $\begin{array}{l}\text { Self-reported 'trouble keeping mind on things' } \\
\text { often or almost always }\end{array}$ & 1 & $131(19)$ & $154(17)$ & 0.474 \\
\hline Stroke & 1 & $71(10)$ & $80(9)$ & 0.441 \\
\hline Peripheral artery disease & 1 & $55(8)$ & $16(2)$ & $<0.001$ \\
\hline Coronary artery bypass surgery ${ }^{b}$ & 1 & $9(1)$ & $49(5)$ & $<0.001$ \\
\hline Body mass index $<18.5 \mathrm{~kg} / \mathrm{m}^{2}$ & 1 & $7(1)$ & $6(1)$ & 0.578 \\
\hline Lack of current alcohol consumption & 1 & $447(63)$ & $743(83)$ & $<0.001$ \\
\hline Risk level according to BDRI & & & & $<0.001$ \\
\hline Low & $0-2$ & 415 (59) & $759(85)$ & \\
\hline Moderate & $3-5$ & $282(40)$ & $128(14)$ & \\
\hline High & $\geq 6$ & $7(1)$ & $7(1)$ & \\
\hline Total score, mean (SD) & & $2.20(1.35)$ & $1.55(1.07)$ & $<0.001$ \\
\hline The Dementia Screening Indicator (DSI) & & $n=631$ & $n=831$ & \\
\hline Age of 70 years & 5 & $631(100)$ & $831(100)$ & 1.000 \\
\hline Less than 12 years of education & 9 & $590(94)$ & $644(78)$ & $<0.001$ \\
\hline Body mass index $<18.5 \mathrm{~kg} / \mathrm{m}^{2}$ & 8 & $5(1)$ & $6(1)$ & 1.000 \\
\hline Type 2 diabetes & 3 & $76(12)$ & $138(17)$ & 0.015 \\
\hline Stroke & 6 & $67(11)$ & $77(9)$ & 0.390 \\
\hline Need for help in managing money or medications & 10 & $16(3)$ & $25(3)$ & 0.588 \\
\hline Depressive symptoms ${ }^{c}$ & 6 & $30(5)$ & $76(9)$ & 0.001 \\
\hline Increased risk for dementia & $\geq 22$ & $30(5)$ & $53(6)$ & 0.184 \\
\hline Total score, mean (SD) & & $15.02(3.94)$ & $13.94(5.23)$ & $<0.001$ \\
\hline
\end{tabular}

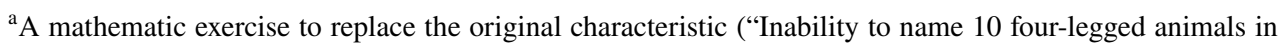
$30 \mathrm{~s}$ ") of the index

${ }^{\mathrm{b}}$ Includes also angioplasty

${ }^{c}$ Use of anti-depressant medications or self-rated feelings of depression age-specific incidence and stable or decreasing age-specific prevalence of dementia in Europe [9-11]. Explanations for the decreasing incidence of dementia are suggested to be higher education $[12,13]$, brain-healthy lifestyle, better treatment of major vascular risk factors $[12,14]$, better access to health care interventions as well as improvements 
in living conditions and social welfare in successive cohorts [15]. Despite time trends in occurrence of dementia, the number of people with dementia is projected to increase mainly because of increased life expectancy and declining rates of mortality [10].

In our study, it was notable that proportions of subjects with an elevated risk for dementia varied a lot based on the index used being distinctly highest according to CAIDE. CAIDE was originally developed for a middle aged population [6], and it showed poor performance in our study which is in line with previous studies also conducted among elderly populations [16-20]. In our study population, all participants received the highest score for age. In addition, CAIDE highlights the role of vascular factors which have found to have inverse associations with dementia among older age groups [20-22]. Therefore, CAIDE is a good index for mid-life dementia risk prediction [23] but application of it among older adults is limited. However, in the Finnish populationbased CAIDE study, a late-life dementia index has recently been developed by using a supervised machine learning method which is able to handle large amounts of data, structure risk factors into groups and give a comprehensive overview of an individual's predictive profile pointing the most relevant risk factors. This late-life dementia index could be useful for dementia prediction of older adults in research settings [10].

BDRI [7] and DSI [8], used in our study, are validated among older ( $\geq 65$ years of age) population, and could, therefore, be appropriate instruments to identify older subjects with an elevated risk for developing dementia later in life. Both indices are brief and easy to use in primary care settings. BDRI includes a combination of age and cognitive, lifestyle and cardiovascular factors. In BDRI, cognitive items are highlighted [7]. In the study of Pekkala et al. [10], cognitive performance was the most important predictor, more predictive than age or vascular factors, for subsequent dementia according to supervised machine learning method using a large number of heterogeneous factors. DSI, instead, is a combination of demographic, vascular and lifestyle factors, difficulties in instrumental activities of daily living and depressive symptoms [8]. In our study, proportion of subjects with an increased risk for dementia according to DSI was low in both cohorts. This is somehow consistent with relative low percentages of subjects with an increased risk for dementia based on DSI in earlier studies, ranging from 6 to $27 \%$ [8]. It is possible that DSI underestimates dementia risk and thereby misses asymptomatic older adults who should be targeted for cognitive screening.

In our study, slightly modified version of CAIDE and BDRI were used in our study. This may have had an impact on risk classification and total scores of indices as well as on comparison of dementia risk between the cohorts. It is also notable, that cohort comparisons cannot confirm changes in risk factors, but only differences between the cohorts. Longitudinal studies can provide more insight regarding changes in dementia risk and cognitive functioning over time.

Our next step is to compare dementia incidence between these two Finnish cohorts as well as to evaluate the prognostic value of BDRI among the cohorts.

Acknowledgements Open access funding provided by University of Turku (UTU) including Turku University Central Hospital. The study was financially supported by ERVA funding of the city of Turku/Welfare Division, Red Feather Campaign of the Nordic Lions Club, and King Gustaf V:s and Queen Victorias Freemasons' Foundation. We would also like to show our gratitude to research nurse Sanna Himanen, who participated in the data collection.

\section{Compliance with ethical standards}

Conflict of interest The authors declare that they have no conflict of interest.

Human and animal rights All procedures performed in studies involving human participants were in accordance with the ethical standards of the institutional or national research committee and with the 1964 Helsinki declaration and its later amendments or comparable ethical standards. This article does not contain any studies with animals performed by any of the authors.

Informed consent Informed consent was obtained from all individual participants included in the study.

Open Access This article is distributed under the terms of the Creative Commons Attribution 4.0 International License (http://creativeco mmons.org/licenses/by/4.0/), which permits unrestricted use, distribution, and reproduction in any medium, provided you give appropriate credit to the original author(s) and the source, provide a link to the Creative Commons license, and indicate if changes were made.

\section{References}

1. WHO (2015) First WHO Ministerial Conference on Global Action against Dementia. https://www.who.int/mediacentre/events/meeti ngs/2015/global-action-against-dementia/en/. Accessed 17 Sept 2018

2. Ngandu T, Lehtisalo J, Solomon A et al (2015) A 2 year multidomain intervention of diet, exercise, cognitive training, and vascular risk monitoring versus control to prevent cognitive decline in at-risk elderly people (FINGER): a randomised controlled study. Lancet 385:2255-2263

3. Rosenberg A, Ngandu T, Rusanen M et al (2018) Multidomain lifestyle intervention benefits a large elderly population at risk for cognitive decline and dementia regardless of baseline characteristics: the FINGER trial. Alzheimers Dement 14:263-270

4. Barnes DE, Yaffe K (2011) The projected impact of risk factor reduction on Alzheimer's disease prevalence. Lancet Neurol 10:819-828

5. Upmeier E, Vire J, Korhonen MJ et al (2016) Cardiovascular risk profile and use of statins at the age of 70 years: a comparison of two Finnish birth cohorts born 20 years apart. Age Ageing 45:84-90 
6. Kivipelto M, Ngandu T, Laatikainen T et al (2006) Risk score for the prediction of dementia risk in 20 years among middle aged people: a longitudinal, population-based study. Lancet Neurol 5:735-741

7. Barnes DE, Covinsky KE, Whitmer RA et al (2010) Dementia risk indices: a framework for identifying individuals with a high dementia risk. Alzheimers Dement 6:138-141

8. Barnes DE, Beiser AS, Lee A et al (2014) Development and validation of a brief dementia screening indicator for primary care. Alzheimers Dement 10:656-665

9. Winblad B, Amouyel P, Andriev S et al (2016) Defeating Alzheimer's disease and other dementias: a priority for European science and society. Lancet Neurol 15:455-532

10. Ahmadi-Abhari S, Guzman-Castillo M, Bandosz P et al (2017) Temporal trend in dementia incidence since 2020 and projections for prevalence in England and Wales to 2040: modelling study. BMJ 358:2856. https://doi.org/10.1136/bmj.j2856

11. Qiu C, Fratiglioni L (2018) Aging without dementia is achievable: current evidence from epidemiological research. J Alzheimers Dis 62:933-942

12. Satizabal CL, Beiser AS, Chouraki V et al (2016) Incidence of dementia over three decades in the Framingham Heart Study. N Engl J Med 374:523-532

13. Noble JM, Schupf N, Manly JJ et al (2017) Secular trends in the incidence of dementia in a multi-ethnic community. J Alzheimers Dis 60:1065-1075

14. Schrijvers EM, Verhaaren BF, Koudstaal PJ et al (2012) Is dementia incidence declining?: trends in dementia incidence since 1990 in the Rotterdam Study. Neurology 78:1456-1463

15. Wu Y-T, Beiser AS, Breteler MB et al (2017) The changing prevalence and incidence of dementia over time-current evidence. Nat Rev Neurol 13:327-339

16. Licher S, Yilmaz P, Leening MJG et al (2018) External validation of four prediction models for use in the general community-dwelling population: a comparative analysis from the Rotterdam Study. Eur J Epidemiol 33:645-655

17. Anstey KJ, Cherbuin N, Herath PM et al (2014) A self-report risk index to predict occurrence of dementia in three independent cohorts of older adults: the ANU-ADRI. PLoS One 9:e86141

18. Hooshmand B, Polvikoski T, Kivipelto M et al (2018) CAIDE dementia risk score, Alzheimer and cerebrovascular pathology: a population-based autopsy study. J Intern Med 283:597-603

19. Solomon A, Soininen H (2015) Dementia: risk prediction models in dementia prevention. Nat Rev Neurol 11:375-377

20. Tang EY, Harrison SL, Errington L et al (2015) Current developments in dementia risk prediction modelling: an updated systematic review. PLoS One 10:e0136181

21. Tolppanen AM, Ngandu T, Kåreholt I et al (2014) Midlife and late-life body mass index and late-life dementia: results from a prospective population-based cohort. J Alzheimers Dis 38:201-209

22. Mielke MM, Zandi PP, Sjögren M et al (2005) High total cholesterol levels in late life associated with a reduced risk of dementia. Neurology 64:1689-1695

23. Hou X-H, Feng L, Zhang C et al (2018) Models for predicting risk of dementia: a systematic review. J Neurol Neurosurg Psychiatry. https://doi.org/10.1136/jnnp-2018-318212

24. Pekkala T, Hall A, Lötjönen J et al (2017) Development of a latelife dementia prediction index with supervised machine learning in the population-based CAIDE study. J Alzheimers Dis 55:1055-1067

Publisher's Note Springer Nature remains neutral with regard to jurisdictional claims in published maps and institutional affiliations. 